\title{
Teneur en acide cholique de quelques biles animales
}

\author{
par C. RICHARD et A. VIALARD-GOUDOU
}

A la suite de demandes d'analyse du Centre de Recherches scientifiques et techniques à Saigon, nous avons été amenés à déterminer la teneur en acide cholique d'extrait mou, de pâte et de poudre de bile de buffle, animal de trait par excellence au Sud-Vietnam.

La section vétérinaire de ce Centre avait entrepris la collecte de biles de buffle et leur concentration en vue d'unc utilisation possible comme matière première pour la préparation de cortisone synthétique.

Par la suite, le Service des Vaccins à l'Institut Pasteur de Saigon nous adressa des échantillons de bile de bufflons vaccinogènes, animaux utilisés pour la préparation du vaccin jennérien. En effet, les milieux de culture préparés à partir de ces biles $n$ 'avaient pas donné satisfaction. On put, après étude, attribuer ces mécomptes à leur teneur très atténuée en acide cholique.

Enfin, le Directeur de l'Institut Pasteur Vétérinaire de Nhatrang, intéressé par notre travail, nous adressa pour détermination du taux d'acide cholique des échantillons de bile de provenances diverses.

En dépit de recherches bibliographiques, et en particulier dans les Chemical Abstracts américains (années 1940-1952), nous n'avons pu trouver, dans la littérature spécialisée, de renseignements concernant les teneurs en acide cholique des biles animales, ni comparer nos résultats à ceux d'auteurs français ou étrangers.

Nous avons divisé notre travail en 2 parties principales :

\section{I. - Biles d'animaux normaux.}

II. - Bile d'animaux ayant servi à la préparation de vaccins humains et vétérinaires.

Dans la première partie, nous indiquons la teneur en acide cholique des biles des animaux suivants : buffles et bufflons, vaches, veaux, bœufs, porcs, porcelets et chiens.

Nous adjoignons à ces premiers chiffres les teneurs en acide cholique de concentrés de bile de buffle et de chien.
Et pour finir cette première partie, nous montrons l'influence de la'stérilisation et du stockage sur la teneur en acide cholique des biles de buffle et de porc.

Dans la seconde partie de cet article, nous examinons les variations de la teneur en acide cholique des biles de bufflons réfractaires ou réceptifs à la maladie vaccinale, de buffles, de bufflons et de veaux " passages" de peste bovine, et enfin de porcs et porcelets «passages » de peste porcine.

Pour terminer, nous signalons, comme précédemment, l'influence de la stérilisation et du stockage sur la teneur en acide cholique de la bile de bufflons vaccinogènes.

Pour toutes ces déterminations, nous, avons utilisé la technique de dosage de l'acide cholique dans les extraits de bile de bcuf, décrite dans la 14 e édition de la Pharmacopée américaine à la pa'ge 410. Nous avons apporté au protocole opératoire quelques modifications que nous signalons plus loin.

\section{TECHNIQUE ANALYTIQUE}

\section{Principe.}

La méthode du codex américain est fondée sur la coloration violette que donne l'acide cholique en milieu acétique avec le furfurol, après addition d'acide sulfurique.

On compare les colorations obtenues'à celle d'une solution d'acide cholique standard de référence traitée dans les mêmes conditions.

\section{Modifications apportées à la méthode américaine.}

a) Une première modification porte sur le poids de la prise d'essai de bile ou de préparation biliée. Il varie suivant l'espèce envisagée ainsi qu'il est indiqué dans le mode opératoire.

b) Enfin nous avons utilisé pour les lectures photométriques l'électrophotomètre de Meunier avec filtre jaune (la longueur d'ondes recommandée est égale à 660 millimicrons). 


\section{Réactifs.}

$1^{\circ}$ Solution acétique.

Acide acétique glacial.... $\quad 600 \mathrm{~cm}^{3}$

Eau distillée Q.S.P. . . . . $1.000 \mathrm{~cm}^{3}$

$2^{\circ}$ Solution de référence d'acide cholique à $50 \mathrm{mg}$ pour $100 \mathrm{~cm}^{3}$.

Peser à la balance de précision $50 \mathrm{mg}$ d'acide cholique chimiquement pur; les dissoudre dans une quantité suffisante d'acide acétique à $60 \%$ pour faire $100 \mathrm{~cm}^{3} ;$ bien mélanger. Chaque $\mathrm{cm}^{3}$ contient $0,5 \mathrm{mg}$ d'acide cholique.

$\mathrm{Si}$ cette solution est conservée au réfrigérateur, on peut l'utiliser pendant de nombreux mois.

\section{$3^{\circ}$ Solution sulfurique.}

Acide sulfurique pur....... $50 \mathrm{~cm}^{3}$

Eau distillée $\ldots . \ldots \ldots \ldots \ldots .65 \mathrm{~cm}^{3}$

Verser lentement l'acide sulfurique dans l'eau, à la température la plus basse possible.

\section{$4^{\circ}$ Solution aqueuse de furfurol à $1 \%$.}

A ne préparer qu'extemporanément.

N'utiliser que du furfurol incolore ou très légèrement coloré.

\section{Mode opératoire :}

Pcscr à la balance de précision, suivant les cas :

$0.1 \mathrm{~g}$ de bile pâte ou de bile poudre;

$0,2 \mathrm{~g}$ d'extrait mou de bile;

I g de bile de chien;

$2 \mathrm{~g}$ de bile de buffle, vache, veau ou bœuf;

$4 \mathrm{~g}$ de bile de buffle ou de veau "passage » de peste bovine;

$5 \mathrm{~g}$ de bile de porc normal ou " passage » de peste porcine, ou de bufflon vaccinogène.

Dissoudre la prise d'essai dans $50 \mathrm{~cm}^{3}$ d'acide acétique à $60 \%$. Filtrer la solution dans une fiole jaugée de $100 \mathrm{~cm}^{3}$. Laver le récipient dans lequel on a pesé la bile, et le filtre, avec de petites portions d'acide acétique à $60 \%$. Compléter à $100 \mathrm{~cm}^{3}$ avec l'acide acétique à $60 \%$ et bien mélanger.

Dans une seconde fiole jaugée de $100 \mathrm{~cm}^{3}$ transvaser $50 \mathrm{~cm}^{3}$ de cette solution, exactement mesurés à la pipette, compléter à $100 \mathrm{~cm}^{3}$ avec de l'acide acétique à $60 \%$. Agiter.

Prélever dans 3 tubes à essai identiques : $1 \mathrm{~cm}^{3}$ de solution d'acide cholique de référence (réactif $n^{0}$ 2), $1 \mathrm{~cm}^{3}$ de la deuxième dilution de bile et $1 \mathrm{~cm}^{3} \mathrm{~d}$ 'acide acétique à $60 \%$. Le contenu de ce dernier tube permettra en fin d'opération de déterminer la position zéro du spot du galvanomèlre de l'électrophotomètre de Meunier.

A chaque tube, ajouter $1 \mathrm{~cm}^{3}$ de solution aqueuse de furfurol à $1 \%$ frâichement préparée et placer immédiatement les tubes dans la glace fondante pendant cinq minutes, puis verser dans chaque tube à l'aide d'une burette, $13 \mathrm{~cm}^{3}$ exactement de solution sulfurique (réactif no 3 ).

Agiter soigneusement. Maintenir ensuite les tubes pendant dix minutes dans un bain-marie réglé à $70^{\circ}$.

Ensuite les plonger pendant deux minutes dans la glace fondante.

Terminer en comparant à l'électrophotomètre de Meunier (filtre jaune) les colorations obtenues pour l'échantillon et le témoin.

Calculs : Soit $\mathrm{T}$ (témoin) et $\mathrm{E}$ (échantillon) les nombres de divisions dont il faut tourner le tambour de l'électrophotomètre de Meunier pour ramener le spot du galvanomètre au zéro.

Si l'on désigne la prise d'essai initiale, mesurée en grammes par $p$, la quantité en milligrammes d'acide cholique par gramme de bile ou de préparation biliée est donnée par la formule.

$$
\frac{100 \times \mathrm{E}}{\mathrm{T} \times P}
$$

En effet, les $p$ grammes de la prise d'essai sont dissous dans $100 \mathrm{~cm}^{3}$ de solution acétique, on en prélève $50 \mathrm{~cm}^{3}$ contenant $\frac{P}{2}$ grammes de bile que l'on étend à $100 \mathrm{~cm}^{3}$.

On effectue la réaction colorée sur $1 \mathrm{~cm}^{3}$ de cette dernière dilution, correspondant par conséquent à $\frac{p}{200}$ grammes de bile.

Parallèlement $1 \mathrm{~cm}^{3}$ de solution de référence contient 0,5 milligramme d'acide cholique.

Or, 0,5 milligramme d'acide cholique correspond à $\mathrm{T}$ divisions de tambour et l'acide cholique contenu dans $\frac{P}{200}$ grammes de bile ou de préparation biliée nécessite $\mathrm{E}$ divisions de tambour.

Il y a donc danis $\frac{P}{200}$ grammes de bile ou de préparation biliée $\frac{0,5 \times E}{T}$ milligrammes d'acide cholique et dans $1 \mathrm{~g}$ de bile ou de préparation biliée :

$$
\begin{aligned}
& \frac{0,5 \times E}{-5 \times \frac{p}{200}}=\frac{0,5 \times 200 \times E}{\mathrm{~T} \times p} \\
& \mathrm{~T} \times \frac{100 \times E}{\mathrm{~T} \times p} .
\end{aligned}
$$

Résultats : Les résultats sont exprimés en miiiìgrammes d'acide cholique par gramme de bile ou de préparation biliée.

Il s'agit en fait des teneurs moyemes en acide cholique des biles et préparations biliées. Chacune de ces moyennes a été calculée après un nombre d'examens indiqué entre parenthèses. 


\section{I. - BILES ANIMALES NORMALES}

\section{a) Biles animales.}

Elles ont été prélevées post-mortem, aussitôt après le sacrifice des animaux.

Les biles de bovins, bubalins et porcins proviennent des abattoirs de Saigon' et de Nhatrang.

Les biles de chien de la fourrière de SaigonCholon.

Leurs teneurs moyennes en acide cholique sont groupées dans le tableau no 1 .

\section{TABLEAU No 1}

Teneur moyenne des biles animales en milligrammes d'acide cholique par gramme

\begin{tabular}{|l|r|r|}
\hline Buffles et Bufflons ... & \multicolumn{1}{|c|}{30,1} & 18 examens \\
Vaches. . . . . . . . & \multicolumn{1}{|c|}{27} & 5 examens \\
Veaux . . . . . . . . & 26,6 & 6 examens \\
Bceufs ... . . . . . & 26,9 & 3 examens \\
Porcs et porcelets. . & 9,1 & 15 examens \\
Chiens. ........... & 47,6 & 12 examens \\
\hline
\end{tabular}

On remarque que le chien, animal carnivore, a une teneur de la bile en acide cholique plus élevée que les bubalins et bovins, animaux herbivores et surtout que les porcins, omnivores, dont les biles ont un taux d'acide cholique particulièrement faible.

\section{b) Concentrés de biles animales.}

Les divers concentrés de bile (extrait mou, pâte et poudre) soumis à notre examen ont été préparés par déshydratation à chaud, en utilisant un dessicateur industriel Maxei.

Ces recherches avaient pour but de déterminer si la teneur de ces produits en acide cholique était suffisante pour qu'on put les exporter en vue de la préparation de cortisone synthétique.

Nos résultats sont condensés dans le tableau no 2.

TABLEAU No 2

Teneur moyenne de concentrés de biles animales en milligrammes d'acide cholique par gramme

\begin{tabular}{|c|c|c|c|c|}
\hline & $\begin{array}{c}\text { BILE } \\
\text { normale }\end{array}$ & $\begin{array}{c}\text { EXTRAIT } \\
\text { mou de bile }\end{array}$ & $\begin{array}{l}\text { BLLE } \\
\text { pâte }\end{array}$ & $\begin{array}{l}\text { BILE } \\
\text { poudre }\end{array}$ \\
\hline $\begin{array}{l}\text { Buffle.. } \\
\text { Chien. }\end{array}$ & $\begin{array}{c}30,1 \\
(18 \text { ex.) } \\
47,6 \\
(12 \text { ex.) }\end{array}$ & $\begin{array}{c}160 \\
(2 \mathrm{ex})\end{array}$ & $\begin{array}{c}275,25 \\
(3 \text { ex.) } \\
382,4 \\
(2 \text { ex.) }\end{array}$ & $\begin{array}{c}399 \\
(3 \text { ex. })\end{array}$ \\
\hline
\end{tabular}

La teneur en acide cholique des concentrés de bile de buffle fut jugée trop faible en France pour l'utilisation envisagée.

Signalons en passant que nous eûmes récemment à examiner des calculs biliaires de bouf, importés de France au Vietnam pour les besoins de la pharmacopée locale sino-vietnamienne.

Cet échantillon titrait 162 milligrammes d'acide cholique par gramme de calcul biliaire.

c) Influence de la stérilisation et du stockage sur la teneur en acide cholique de 2 biles animales.

Nous avons déterminé la teneur en acide cholique d'une bile de buffle et d'une bile de porc, toutes deux recueillies post-mortem.

Ces échantillons ont été mis en ampoule scellée et autoclavés pendant une demi-heure à la température de 115 degrés.

Après une épreuve de conservation de vingt jours, nous avons procédé à nouveau au titrage de l'acıde cholique.

Les résultats obtenus sont rapportés dans le Tableau no 3 .

\section{TABBLEAU No 3}

Titre en acide cholique de biles animales avant et après stérilisation et stockage, en milligrammes d'acide cholique par gramme.

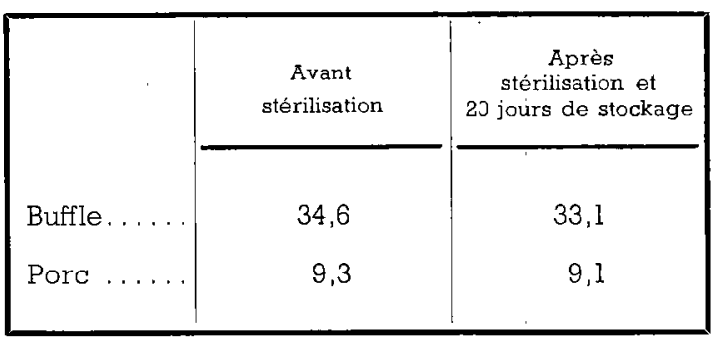

On voit donc que la stérilisation et le stockage n'affectent pratiquement pas le titre en acide cholique des bilos oxaminćes.

\section{II. - VRRIATION DU TAUX D'BCIDE CHO- LIQUE DES BIIES D'ANIMAUX \& PASSAGES 》 AYANT SERVI A LA PRÉPARATION DE VACCINS}

Ties biles de bufflons vaccinogènes proviennent de l'Institut Pasteur de Saigon, celles de buffles et de veaux «passages » de peste bovine, et celles de porcs et porcelets " passages » de peste porcine, de l'Institut Pasteur Vétérinaire de Nhatrang.

Le tableau $n^{\circ} 4$ fait apparaître les modifications que nous avons constatées: 


\section{TABLEAU No $\quad 4$}

Teneur en acide cholique des biles d'animaux ayant servi à la préparation de vaccins humains et vétérinaires, en nilligrammes d'acide cholique par gramme.

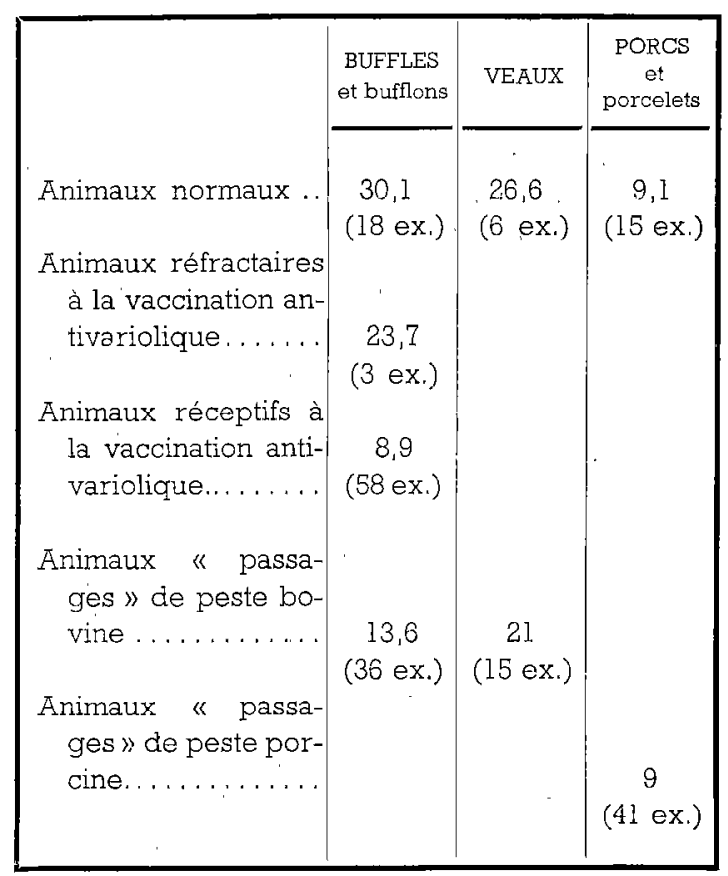

La lecture du tableau no 4 montre que les bubalins utilisés à la préparation du vaccin antivariolique humain ou servant de "passages » à la peste bovine ont des taux d'acide cholique dans la bile nettement abaissés.

Par contre, la teneur en acide cholique de la bile des veaux servant de passage à la peste bovine n'est que faiblement modifiée, celle des porcins « passages » de peste porcine reste inchangée.

Enfin' la stérilisation et le stockage en ampoule scellée d'un échantillon de bile de bufflon vaccinogène n'apportent aucune modification sensible à la teneur en acide cholique (5,2 avant et 5,1 après stérilisation et stockage).

\section{RÉSUMÉ}

L'étude des teneurs moyennes en acide cholique par gramme de bile de quelques animaux a donné les résultats suivants :

Buffles et bufflons : 30, I mg. - Vaches : $27 \mathrm{mg}$. Veaux : 26,6 mg, - Bceufs : 26,9 mg. - Porcs et porcelets : $9,1 \mathrm{mg}$. - Chiens : $47,6 \mathrm{mg}$.

Lors de la préparation des vaccins antivariolique humain et antipestique bovin, cette teneur chez les bubalins est sénsiblenilent abaissée ( 8,9 et 13,6 mg), par contre les modifications constatées chez les bovins et les porcins ayant servi à la préparation de vaccins antipestiques bovin et porcin sont peu significatives pour les premiers et nulles pour les seconds. La stérilisation et le stockage ne' modifient sensiblement pas les teneur's des biles animales en acide cholique. Enfin dans des concentrés de bile de buffle nous avons pu trouver jusqu'à $400 \mathrm{mg}$ d'acide cholique par gramme.

(Service de Chimie de I'Institut Pastcur de Saigon.) 